\title{
Methode zur Abscheidung der Phosphorsäure bei der qualitativen Analyse.
}

\author{
Fon D. Batarew. $\left.{ }^{1}\right)$
}

Zur Abscheidung der Phosphorsäure aus der dritten Gruppe der Kationen, kann die geringe Löslichkeit des Bleiphosphats in verdünnter Salpetersäure, bzw. Essigsäure, verwendet werden.

\section{Methode zur Abscheidung.}

Nachdem man aus der salzsauren Lösung der Fällung der Schwefelammoniumgruppe (die also schon frei von $\mathrm{Co}$ und $\mathrm{Ni}$ ist) den Schwefelwasserstoff entfernt hat, tropft man so lange verdünnte $\mathrm{NH}_{3}$ dazu (Bemerkung 1), bis ein beständiger Niederschlag entstanden ist. (Einen kleinen Teil der Lösung behält man zurück.) Man löst den Niederschlag in möglichst wenig $\mathrm{HCl}$, und fügt $\mathrm{PbNO}_{3}$-Lösung in großem Überschuß dazu (Bemerkung 2). Weiter gibt man einige Tropfen Methylorange und unter ständìgem Umrühren Natriumacetatlösung dazu, bis die rote Färbung der Lösung zu verschwinden beginnt. Nach ungefähr 5 Minuten anhaltenden Umrübrens filtriert man den entstandenen Niederschlag von $\mathrm{PbCl}_{2}$ und $\mathrm{Pb}_{3}\left(\mathrm{PO}_{4}\right)_{2}$ ab (Bemerkung 3). Obgleich nun $\mathrm{FePO}_{4}, \mathrm{AlPO}_{4}$ und $\mathrm{CrPO}_{4}$ in Essigsäure unlöslich sind, fällt bei dem großen ÜberschuB an $\mathrm{Pb}$-Kationen die Phosphorsäure zum gröBten Teil als Bleiphosphat aus und im Filtrate bleiben immer noch kleine Mengen von $\mathrm{Fe}^{\cdots}$, $\mathrm{Cr} \cdots$ und $\mathrm{Al}^{\cdots}$ zurück, die zum sicheren Nachweis genügen. Das Filtrat wird mit Ammonium-Molybdatlösüng auf Phosphorsäure geprüft. Falls die Säure noch nicht vollständig abgeschieden ist, fügt man noch einige Tropfen Acetatlösung hinzu.

Aus dem Filtrate entfernt man nun zuerst das überschüssige Blei mit $\mathrm{HCl}$ (Bemerkung 4) und trennt dann die dritte und vierte Gruppe durch $\left(\mathrm{NH}_{42}\right)_{2} \mathrm{~S}$ bzw. $\mathrm{NH}_{4} \mathrm{Cl}$ und $\left(\mathrm{NH}_{4}\right)_{2} \mathrm{~S}$. Der Niederschlag von Gruppe III wird immer durch $\mathrm{PbS}$ schwarz gefärbt sein, aber dieses

1) Bei der Ausführung dieser Arbeit waren mir die Herren G. KandiLarow and N. Pentschew behilflich. Ich spreche ihnen hiermit meinen Dank aus. 
Sulfid bleibt beim Auflösen der Hydrate und Sulfide der dritten Gruppe in verdünnte $\mathrm{H}_{2} \mathrm{SO}_{4}$ als unlöslich zurück (Bemerkung 5).

Auf diese Weise erhalten wir Filtrate der dritten und vierten Gruppe, die frei von Phosphorsäure und Blei sind (Bemerkung 6).

\section{Analytische Belege.}

Es wurde nach der oben angegebenen Methode zuerst eine Mischung, die ca. 0,01 g Al, Cr, $\mathrm{Zn}, \mathrm{Fe}, \mathrm{Mn}, \mathrm{Ba}, \mathrm{Sr}, \mathrm{Ca}, \mathrm{Mg}$ und verschiedene Mengen $\mathrm{H}_{3} \mathrm{PO}_{4}(0,5-5 \mathrm{~g})$ enthielt, analysiert. Allo Kationen wurden einwandsfrei nachgewiesen.

Außerdem wurde zu den Lösungen der einzelnen Salze der anderen Metalle der dritten Gruppe Phosphorsäure hinzugefügt, und dann die letztere nach der oben angegebenen Bleimethode abgeschieden. Es zeigte sich, daß Titan, Uran und Zirkonium mit dem Bleiphosphat zusammen ausfallen. Nachdem man aber den Niederschlag mit $\mathrm{H}_{2} \mathrm{SO}_{4}$ ausgezogen hat, kann man leicht die genannten Elemente in dem Filtrat nachweisen.

\section{Vorzüge der von mir angegebenen Bleimethode.}

Die oben angegebene Bleimethode zur Abscheidung der Phosphorsäure aus der dritten analytischen Gruppe der Kationen, hat vor den bis jetzt gebräuchlichen Eisen-, Zinn- und Phosphatmethoden folgende Vorzüge:

Die Phosphorsäure wird schon beim ersten Male entfernt, während bei der Eisen- und Zinnmethode die vollkommene Entfernung erst nach der zweiten und dritten Bearbeitung erreicht ist.

Die Darstellung besonderer Präparate, z. B. Zinnsäure, ist hier nicht nötig.

Alle häufiger vorkommenden Kationen der dritten Gruppe bleiben im Filtrat, während bei der Eisenmethode Al und $\mathrm{Cr}$ mit dem basischen Eisenacetat zusammen ausfallen.

Das AufschlieBen des $\mathrm{BaSO}_{4}$ und $\mathrm{SrSO}_{4}$ ist ein grober Nachteil der Phosphatmethode.

Die Bleimethode führt schneller zam Ziel als die Zinnmethode, weil bei diesen Operationen zur Entfernung des Chlors und zur Beseitigung des $\mathrm{Pb}$ und $\mathrm{Cu}$ und von Spuren der Zinnsäure nötig sind.

Bemerkungen.

1. Man benutzt zum Neutralisieren nicht $\mathrm{NaOH}$, da die Alkalisalze die Kalzinierung des Rückstandes der vierten Gruppe erschweren, die zur Entfernung der Ammoniumsalze notwendig ist. 
2. Der ÜberschuB an Bleinitratlösung muß 5-10 mal so groB sein, als die nach dem Fällen der $\mathrm{Cl}$-Anionen als $\mathrm{PbCl}_{2}$ zum Fällen der Phosphorsäure noch erforderliche Menge. Beim Arbeiten mit weniger Bleinitratlösung fällt das Chrom mit dem Bleiphosphat zusammen aus. Deshalb sucht man das Chrom, falls es in dem Filtrat der dritten Gruppe nicht nachgewiesen werden kann, in dem zurückbehaltenen kleineren Teil der Lösung; die Phosphorsน̆ure stört diesen Nachweis nicht.

3. Oft läuft das Filtrat trübe durch das Filter. Es bandelt sich aber dann nicht um Bleiphosphat, weil dieses stets kristallinisch ansfallt, vielmohr wird die Trübung durch basische Karbonate und Acetate hervorgerufen. Man kann das Filtrat also ungeachtet der Trübung auf Phosphorsäure prüfen.

4. Nicht mit $\mathrm{KCl}$ oder $\mathrm{NaCl}$, siehe Bemerkung 1.

5. Beim Arbeiten mit HCl geht ein Teil des Bleis in Lösung und färbt das $\mathrm{ZnS}$ schwarz.

6. Falls die Menge der Ammoniumsalze im Filtrate der vierten Gruppe sehr groB ist gegenäber dem vorhandenen $\mathrm{Ba}, \mathrm{Sr}$ und $\mathrm{Ca}$, so muB das Filtrat eingedampft und kalziniert werden. Die saure Lösung des kalzinierten Rüekstandes gibt mit $\mathrm{NH}_{3}$ eine amorphe grobflockige Fällung, die in Essigsäure löslich ist. Diese Fällung ist kein $\mathrm{Mg}(\mathrm{OH})_{2}$, sondern besteht wahrscheinlich aus Salzen der Erdalkalien mit Säuren, die durch Oxydation von Essigaäure infolge der Anwesenheit von Nitraten entstanden sind. Da diese Oxydation nur beim Kalzinieren des Rückstandes stattfindet, so darf bei der zweiten Fällung der schon phosphorsäurefreien) Lösung nur ein geringer ÜberschuB von $\left(\mathrm{NH}_{4}\right)_{2} \mathrm{~S}$ zugesetzt werden, um das Kalzinieren des Rückstandes zu vermeiden.

Die in Frage kommende amorphe Fällung bindert jedoch nicht den Nachweis des $\mathrm{Ba}$ und $\mathrm{Sr}$, da dabei die Lösung sauer ist. Erst beim Fällen des Calciums und Magnesiums tritt neben kristallinischem $\mathrm{CaC}_{2} \mathrm{O}_{4}$ und $\mathrm{MgNH}_{4} \mathrm{PO}_{4}$ aq. auch ein amorpher Niederschlag auf, dessen Bildung jedoch vermieden werden kann, wenn man auf folgende Weise verfährt: Man dampft den Rückstand der vierten Gruppe nicht zur Trockne ein, sondern fallt zuerst das Barium als $\mathrm{BaCrO}_{4}$ und kocht das Filtrat in einer Porzellanschale mit kleinen Mengen von $\mathrm{Na}_{8} \mathrm{CO}_{3}$ bis der $\mathrm{NH}_{\mathrm{s}}$-Geruch verschwindet. Die gefällten Karbonate untersucht man auf $\mathrm{Sr}, \mathrm{Ca}$ und $\mathrm{Mg}$. Falls hierbei das Magnesium nicht nachgewiesen wird, sucht man es im Filtrat nach Fällen mit $\mathrm{Na}_{8} \mathrm{CO}_{3}$.

\section{Sofla, Chemisches Institut der Universität.}

Bei der Redaktion eingegangen am 14. November 1921. 